\title{
El grado de competencia mediática en la ciudadanía andaluza
}

\author{
Jacqueline SÁNCHEZ CARRERO \\ Universidad de Huelva \\ jsanchez.carrero@sic.uhu.es \\ José Ignacio AguADED GómEZ \\ Universidad de Huelva \\ vicerrector.tecnologias@uhu.es
}

Recibido: 14/09/2012

Aceptado: 16/01/2013

\begin{abstract}
Resumen
Conocer el grado de competencia mediática de la ciudadanía es hoy un reto crucial para entender la sociedad de la información en la que estamos inmersos y, por ende, una tarea imprescindible para establecer acciones formativas en alfabetización mediática. Este trabajo recoge los resultados globales de investigación obtenidos en una variada muestra de la ciudadanía andaluza y e inserto en un amplio estudio realizado a nivel estatal durante los últimos años con la implicación de las 17 Comunidades Autónomas y abanderado por el Consejo Audiovisual de Cataluña. El estudio define, en primer lugar, el polisémico término competencia mediática para luego segmentarlo en sus seis dimensiones clave: estética, lenguaje, ideología y valores, tecnología, producción y programación, y recepción y audiencia. La presente investigación concluye que la población -que constituye la muestra- carece de competencia mediática en dichas dimensiones, por lo se hace necesaria la integración de una asignatura educación mediática en el currículo escolar.
\end{abstract}

Palabras clave: Competencia, mediática, educación, Andalucía, dimensiones, ciudadanía.

\section{The Extent of Media Competence among Citizens in Andalusia}

\begin{abstract}
Determining the extent of media competence among citizens today is an important challenge if we are to understand the information society in which we live, and formulate educational interventions that foment media literacy. This work analyses the results of research based on a broad sample of citizens in Andalusia which formed part of a wide-ranging study carried out in the past few years in all 17 of Spain's autonomous regions, and which was promoted by the Audiovisual Council of Catalonia. The study defines the polysemantic term media competence and deconstructs it into six dimensions: aesthetics, language, ideology and values, technology, production and programming, audience and reception. The study concludes that the sample population is lacking in media competence in all six dimensions, and urges that media literacy be added to the school curriculum.
\end{abstract}

Keywords: Competition, media, education, Andalusia, dimensions, citizenship.

\section{Referencia normalizada}

SÁNCHEZ CARRERO, Jacqueline y AGUADED GÓMEZ, J. Ignacio (2013): "El grado de competencia mediática en la ciudadanía andaluza". Estudios sobre el mensaje periodístico. Vol. 19, Núm. 1, págs.: 265-280. Madrid, Servicio de Publicaciones de la Universidad Complutense.

Sumario: 1. Competencia mediática: marco teórico; 1.1. Concepto y estudios sobre competencia mediática; 1.2. Justificación: ¿por qué se debe conocer el grado de competencia mediática de una sociedad?. 2. La investigación desde el enfoque cuantitativo; 2.1. Objetivos, metodología y muestra de la investigación; 2.2. El cuestionario; 2.3. Las dimensiones analizadas; 2.3.1. Dimensión estética; 2.3.2. Dimensión de lenguaje; 2.3.3. Dimensión de la ideología y de los valores; 2.3.4. Dimensión de la recepción y la audiencia; 2.3.5. Dimensión de la tecnología; 2.3.6. Dimensión de la producción y la programación. 3. Conclusiones. 4. Agradecimientos y apoyos. 5. Referencias bibliográficas. 


\section{Competencia mediática: marco teórico}

\subsection{Concepto y estudios sobre competencia mediática}

La competencia mediática es un término que viene tomando auge en los últimos tiempos sobre todo en los campos educativos y tecnológicos. Este concepto está estrechamente relacionado con el de competencia en comunicación audiovisual. Pero conviene entender primeramente qué se entiende como competencia. Se conoce como tal a "una combinación de conocimientos, capacidades y actitudes que se consideran necesarios para un determinado contexto" (Ferrés, 2007: 100). Tal como afirma Ferrés, este término ha estado vinculado desde un principio al sector empresarial hasta que comenzó a hacerse un lugar propio en el mundo académico. En la actualidad forma parte de una de las líneas de investigación académica con distintas tendencias: competencia básica -las que ayudan al individuo en su entorno social-, competencia lingüística -la que desarrolla un sujeto para comprender y expresarse en una lengua determinada-, competencias en educación, entre otras.

En este caso se habla del campo audiovisual en concreto, por lo tanto el concepto hace referencia a esos conocimientos, capacidades y actitudes con las que cuenta un sujeto en lo relacionado con el mundo audiovisual. Esa capacidad del individuo para codificar y decodificar los mensajes audiovisuales se ha venido estudiando desde hace más de tres décadas, pero no es sino hasta estos últimos años, específicamente desde que el Parlamento Europeo define lo que es la educación mediática, cuando comienza a aceptarse a la alfabetización audiovisual como una alfabetización tan importante como la que logra el niño en sus primeras etapas de vida escolar. Solo que no se trata de aprender a leer y a escribir en el papel sino que lo hace en el mundo digital, rodeado de pantallas, tal como el que vivimos en el siglo XXI. No obstante, la competencia audiovisual es un campo poco estudiado. No son muchos los trabajos que indagan e interiorizan en la actitud de una persona para conocer su nivel de entendimiento y comprensión -desde todas las perspectivas posibles- del medio audiovisual, sobre todo en el caso de los niños y adolescentes. Son éstos precisamente quienes tienen un mayor riesgo puesto que comparte información en el medio online y manejan -a su manera- diversos medios digitales como Internet y la telefonía móvil, cuyo desarrollo llega a ser vertiginoso.

Las recomendaciones que el Parlamento Europeo redactó en 2006 sobre competencias clave para el aprendizaje permanente han servido de base para el mundo de la educación. Desde entonces el sector puede contar con un marco de referencia europeo y así definir sus líneas acordes con las nuevas cualificaciones básicas que debe proporcionar el aprendizaje permanente. El documento destaca un total de ocho competencias clave, a saber: comunicación en la lengua materna; comunicación en lenguas extranjeras; competencia matemática y competencias básicas en ciencia y tecnología; competencia digital; aprender a aprender; competencias sociales y cívicas; sentido de la iniciativa y espíritu de empresa; y conciencia y expresión culturales. La competencia digital dirige la acción hacia "el uso seguro y crítico de las tecnologías de la sociedad de la información para el trabajo, el ocio y la comunicación"”. Aunque la

1 Cfr. Las recomendaciones completas pueden ser leídas en Recomendación del Parlamento Europeo y del Consejo de 18 de diciembre de 2006 sobre las competencias clave para el 
competencia digital no abarca lo estrictamente audiovisual, sí guarda relación y sirve de plataforma para la investigación. Se puede afirmar que la competencia en comunicación hace referencia más bien a los conocimientos, habilidades, capacidades, valores y actitudes, que un sujeto tiene ante el medio audiovisual.

Pero no ha sido el Parlamento Europeo la única institución que ha realizado propuestas concretas en los últimos años acerca de la educación en comunicación audiovisual $^{2}$. Mucho antes, a inicios de los ochenta, ya se conocía la Declaración de Grünwald, promulgada por la UNESCO, en la cual se invitaba a fomentar la educación hacia la comprensión crítica de la comunicación en la ciudadanía. Algunos otros organismos internacionales como la ONU, a través de Alianza para las Civilizaciones, están llevando a la práctica acciones concretas. En este caso lo han logrado a través del diseño de herramientas como el Kit de Educación en Medios y el Currículo de formación del educador en medios y alfabetización comunicativa. Otros, han optado por aunar fuerzas para publicar documentos suscritos por distintas instituciones, desde mediados de esta década, como ejemplo pueden ser citados: Alfabetización Informacional y el Aprendizaje de por vida (Proclamación de Alejandría, 2005), la Agenda de París para la Educación en Medios (2007), la Conferencia de Oriente Medio sobre la Educación en Medios en Arabia Saudita (2007), el Foro Internacional de Investigación sobre Medios (Londres; Hong Kong, 2008), la Conferencia en Nigeria sobre Medios en África (2008), entre otros como las Recomendaciones del Parlamento Europeo. En el II Congreso Media Literacy in Europe controversies, challenges and perspectives (Italia, 2009) se llevaron a la mesa de discusión aportaciones de relevantes investigadores europeos como Buckingham, Tisseron, Bazalgette, Carlsson, Bevort, Verniers o Piette, quienes debatieron y señalaron a la competencia mediática como una competencia básica que ha de adquirir necesariamente la ciudadanía.

Al hilo de lo expuesto se puede observar que en materia de publicaciones hay una mayor presencia en el universo digital, se ha avanzado mucho en comparación con aquellos últimos años del siglo XX. Un modelo a señalar es el de Alianza de Civilizaciones $(\mathrm{ONU})^{3}$, que dispone de un portal de información -un repositorio online- especializado en la educación mediática vinculada con temas de actual importancia tales como la migración, la igualdad, la ecología mediática y las nuevas iniciativas educativas. Esta iniciativa facilita la aportación de los investigadores a un archivo centralizado y clasificado por temas, idiomas, país y tipo de información.

aprendizaje permanente (2006/962/CE). En ella destacan además los objetivos y la definición de cada una de las ocho competencias para el aprendizaje a lo largo de la vida. http://eurlex.europa.eu/LexUriServ/LexUriServ.do?uri=CELEX:32006H0962:ES:NOT

2 Cfr. Aparte de las Recomendaciones de 2006 el Parlamento Europeo publicó en 2008 el documento Un planteamiento europeo de la alfabetización mediática en el entorno digital, en él se instaban a las autoridades a promover la alfabetización mediática y elaborar códigos de conducta junto con las organizaciones pertinentes para promover la actividad en este campo. http://eur-lex.europa.eu/smartapi/cgi/sga_doc?smartapi!celexplus!prod!DocNumber $\& \lg =$ es\&type_doc $=$ COMfinal\&an_doc $=2007 \& n u \_d o c=833$

3 Cfr. El portal de la Alianza de la Civilizaciones, versión en español, puede consultarse en: www.aocmedialiteracy.org/index.php?lang=spanish 


\subsection{Justificación: ¿por qué se debe conocer el grado de competencia mediática de una sociedad?}

Si bien es cierto que se ha constituido una especie de conciencia sobre la necesidad de la educación mediática a nivel internacional no es menos real que hay que dar el paso siguiente a las recomendaciones, definiciones y consejos por parte de las más destacadas instituciones. No son muchos los programas de educación mediática que son reconocidos como parte del currículo en la escuela aún cuando en algunos casos los ofrecen como materia optativa, en la cual el estudiante puede escogerla o decantarse por otra. Urge pasar a la práctica. Las razones por las que se debe conocer el grado de competencia mediática de la ciudadanía son varias y diversas pero todas se centran en conocer qué propuestas formativas se han de aplicar para llevar a cabo una educación mediática efectiva. Ya en 2007 en la Conferencia Internacional de la UNESCO en Paris se hablaba de las dificultades de implementar una alfabetización mediática integrada en el sistema escolar. Piette tiene razón cuando afirma que algunas de las objeciones que ha encontrado la puesta en marcha de la educación mediática en la mayoría de países son las siguientes: escasa formación inicial del profesorado, confusiones entre educación "por" o "sobre" los medios de comunicación y búsqueda de fórmulas educativas del tipo "listo para enseñar" -de tal modo que solo tenga que reproducir en clase un programa ya probado-, entre otras ${ }^{4}$.

La Comisión Europea en el documento titulado Recomendaciones sobre la alfabetización mediática en el entorno digital para una industria audiovisual y de contenidos más competitiva y una sociedad del conocimiento, insiste en la necesidad de conocer los criterios de evaluación de los niveles de alfabetización mediática en Europa: "fomentar la investigación sistemática mediante estudios y proyectos sobre los diferentes aspectos y dimensiones de la alfabetización mediática en el entorno digital y vigilar y medir el progreso de los niveles de la misma" ". No existen planteamientos sistematizados de evaluación de las competencias en comunicación audiovisual. La formación es insuficiente o inexistente en la escuela y en la Universidad ocurre algo similar, salvo en las carreras que la contienen como eje principal. Hasta que no se desarrollen los procesamientos de evaluación de la actividad no se sabrá efectivamente en qué medida se aplica realmente y si lo que se ofrece es lo adecuado. Pero antes de dicha evaluación conviene conocer cuál es el nivel de comprensión que tiene la ciudadanía a este respecto.

Por ello el Consejo Audiovisual de Cataluña impulsó en el 2005, junto con la Universidad Pompeu Fabra, las acciones que desembocarían en un estudio sobre la competencia mediática en España. A esta convocatoria acudieron representantes de algunas universidades españolas con el fin de participar en la propuesta liderada por Joan Fe-

${ }^{4}$ Crf. El apartado redactado por Jacques Piette titulado The challenges of media literacy abarca otros aspectos tales como que representa una tarea demasiado costosa, la inseguridad del docente o la condición de la educación mediática infantil. Puede leerse en Euromeduc: A European exchange network for media literacy (2009) en: www.euromeduc.eu/IMG/pdf/Euromeduc_ENG.pdf, 115-124.

5 Véase el documento redactado por la Comisión Europea con fecha de 2009 en: http://ec.europa.eu/culture/media/literacy/docs/recom/c_2009_6464_es.pdf; página 6, número I.2. 
rrés, que consistía en medir ese grado de conocimiento de la población en España a través del trabajo por comunidades autónomas. Después de lograr el reconocimiento del proyecto por parte del Ministerio de Educación, el estudio se hizo extensible a diecisiete Universidades de todo el territorio estatal ${ }^{6}$. En agosto de 2011 el Instituto de Tecnologías Educativas (ITE), perteneciente al Ministerio de Educación, publicó el proyecto marco que posibilitó la presente investigación, y que se tituló: Competencia Mediática. Investigación sobre el grado de competencia de la ciudadanía en España (Ferrés y otros, 2011). La conclusión general extraída de los resultados es que: "el grado de competencia mediática de los españoles es muy bajo. La nota media que obtienen las personas de la muestra es un 24,5 sobre 100 , con una desviación típica de 13,44 puntos. El $95,4 \%$ suspende, es decir, obtiene una puntuación inferior al 50\% del total de los valores asignados. La nota máxima conseguida, además, también es muy baja, puesto que es de 84 puntos, que obtiene el 0,06\% de las personas de la muestra"7. En este estudio se aplicó una metodología cuantitativa-cualitativa produciéndose más de una veintena de conclusiones. Se destacan la síntesis de siete de ellas ${ }^{8}$ :

1. Se detectan diferencias significativas en cuanto al déficit de sentido crítico en función de la variable edad.

2. Algunas personas consideran que en los mensajes mediáticos solo hay ideología y valores cuando hay intencionalidad de transmitirlos por parte de los autores. Otras no son conscientes de la presencia de estereotipos y de valores si éstos son latentes. Hay otras que, siendo conscientes de la presencia, parece no importarles o no molestarles.

3. En esta investigación cualitativa se confirman también los resultados de la cuantitativa en cuanto al desconocimiento sobre el porqué y el cómo puede influir un mensaje cuando no utiliza ningún tipo de argumento.

4. Entre las personas de la muestra se detecta una preocupación grande por la objetividad de la información mediática, así como la certeza de que hace falta confrontar las informaciones para poder tener garantías respecto a su fiabilidad.

5. Abundan los casos de personas que no demuestran tener unos criterios sólidos a la hora de elegir las cadenas de televisión que utilizan para informarse.

${ }^{6}$ Crf. Esas Universidades fueron: Cataluña (Universidad Pompeu Fabra); Andalucía (Universidades de Huelva, Granada, Málaga, Cádiz, Córdoba, Sevilla); Navarra (Universidad de Navarra); País Vasco (Universidad del País Vasco); Madrid (Universidades Carlos III y Complutense); Galicia (Universidad de Santiago); Aragón (Gobierno de Aragón); Valencia (Universidad Jaume I); Asturias (Gobierno de Asturias); Cantabria (Universidad de Cantabria); Murcia (Universidad Católica de Murcia); Canarias (Universidad de La Laguna); La Rioja (Universidad de La Rioja); Castilla-La Mancha (Universidad de Castilla-La Mancha); Castilla-León (Universidad de Valladolid); Baleares (Universidad Islas Baleares); y Extremadura (Universidad de Extremadura).

7 El análisis de los resultados totales puede ser consultado en: http://ntic.educacion.es/w3 //competencia_mediatica/2_evaluacion/7.htm.

8 Las conclusiones en su totalidad y las propuestas de actuación pueden leerse en: Competencia Mediática. Investigación sobre el grado de competencia de la ciudadanía en España (Ferrés y otros, 2011) http://ntic.educacion.es/w3//competencia_mediatica/3_evaluacion/7.htm 
6. No es demasiado elevado el nivel de conciencia sobre los condicionamientos a los que están sometidas las cadenas y las otras instituciones que trabajan en el mundo informativo.

7. En general se constata que las redes tienen un inmenso potencial para promover la creatividad y la interacción y, sin embargo, el sistema educativo no promueve de manera suficiente el ejercicio de un pensamiento crítico estimulador de la creatividad.

Como se puede observar estas premisas suman un conglomerado muy interesante a la hora de analizarlas y preparar propuestas de formación pues abarcan campos de actuación muy diversos y multidisciplinares. Lo que a continuación se describe es el estudio cuantitativo realizado en Andalucía, el cual estuvo a cargo del Grupo de Investigación Ágora de la Universidad de Huelva y del Grupo Comunicar?.

\section{La investigación desde el enfoque cuantitativo}

\subsection{Objetivos, metodología y muestra de la investigación}

Para medir el grado de competencia audiovisual de la ciudadanía andaluza el grupo acordó alcanzar los siguientes objetivos:

- Detectar las necesidades y carencias de la población andaluza seleccionada en relación con los niveles de competencia en comunicación: tecnología, lectura crítica y producción audiovisual.

- Definir los indicadores de referencia para medir el grado de competencia en comunicación en sectores poblacionales diferenciados de todas las provincias de Andalucía.

- Diagnosticar los niveles y grado de competencia audiovisual de las muestras seleccionadas.

En otros estudios ya aprobados de I+D y Proyecto de Excelencia, este grupo de investigación estudia las "buenas prácticas" en alfabetización y contenidos de ocio digital. Todo esto con la misma finalidad de diseñar propuestas de acción formativas en el campo digital y audiovisual que puedan servir a padres y maestros para la práctica de la educación mediática ${ }^{10}$.

${ }_{9}$ Cfr. El equipo final estuvo constituido por Ignacio Aguaded (IP), Joan Ferrés (IP Estatal), $\mathrm{M}^{\mathrm{a}}$ del Rocío Cruz Díaz, Ma Amor Pérez Rodríguez, Jacqueline Sánchez Carrero y Águeda Delgado. Como colaboradores desplegados por toda la comunidad autónoma andaluza figuran: Almería: Jorge Martínez Muñoz y Concepción Fernández Soto; Cádiz: Francisco Pavón Rabasco y Juan Casanova; Córdoba: Verónica Marín y Antonia Ramírez; Granada: Stella Martínez y Antonio Rodríguez; Huelva: Julio Tello Díaz y Ángel Hernando Gómez; Jaén: Rafael Quintana y Concha Araujo; Málaga: Ana Almansa y Fernando Navarro; y Sevilla: Pedro Román Graván y J. María Batanero.

${ }^{10}$ El Proyecto I+D+i se titula "La competencia en comunicación audiovisual en un entorno digital. Diagnóstico de necesidades en tres ámbitos sociales". El proyecto de Excelencia lleva por nombre "La Competencia audiovisual de la ciudadanía andaluza: estrategias de alfabetización mediática en la sociedad del ocio digital". Ambos se desarrollan durante el trienio 2010-13. 
La muestra ha sido seleccionada aleatoriamente por estratos de cada una de las 8 provincias andaluzas. Las variables han sido edad, sexo y nivel de estudios. Con esta muestra no se pretendía significatividad poblacional. En relación con la metodología utilizada para esta investigación se utilizó la cuantitativa -a través del uso del cuestionario- y la cualitativa -mediante el establecimiento de grupos de discusión y entrevistas en profundidad- la cual se aplicará en una segunda fase de modo de tener una perspectiva más completa de la situación. Por esta razón se describe aquí lo relativo a la primera de ellas, la cuantitativa. En cuanto al universo de estudio estuvo constituido por personas mayores de 16 años residente en la comunidad autónoma de Andalucía. En cuanto a los codificadores fueron docentes e investigadores sometidos previamente a un proceso de formación metodológica y análisis de cuestionarios que les permitió unificar criterios en los distintos aspectos que abarca el cuestionario. Así, en el transcurso de 2011 se aplicaron en total 599 cuestionarios a una muestra que, según las variables escogidas que se muestran en la Tabla 1, presenta las siguientes características:

Tabla 1. Constitución de las variables escogidas para este estudio

\begin{tabular}{|l|l|}
\hline \multirow{2}{*}{ Provincias e integrantes } & $\begin{array}{l}\text { Cádiz 104, Jaén 89; Málaga, Granada y Huelva 78; Almería 77; Cór- } \\
\text { doba 51 y Sevilla 43. }\end{array}$ \\
\hline \multirow{2}{*}{ Género } & $\begin{array}{l}251 \text { hombres } \\
343 \text { mujeres }\end{array}$ \\
\hline \multirow{2}{*}{ Edad } & $\begin{array}{l}\text { Jóvenes (16-24 años) } 21,7 \% ; \\
\text { Adultos (25-64 años) } 61.4 \% ; \\
\text { Tercera edad (a partir de } 65 \text { años) } 13,5 \% .\end{array}$ \\
\hline \multirow{2}{*}{ Nivel de estudios } & $\begin{array}{l}\text { Sin estudios 4,9\%; Con estudios primarios } 17,2 \% ; \\
\text { Con estudios secundarios } 45,4 \% \text {; con estudios superiores } 32,4 \% .\end{array}$ \\
\hline
\end{tabular}

De todos ellos casi la mitad -un $49.7 \%$ que conforma la mayoría- declara no tener ningún tipo de formación en comunicación audiovisual frente a un $32.7 \%$ de ellos considera que tiene cierto grado de formación al respecto. Esta es la situación a pesar de que desde la LOGSE hasta la Ley Orgánica de Educación que rige en este momento se dictaminar la integración transversal de las tecnologías de la información y la comunicación. Lo mismo se indica en cuanto al tratamiento educativo de los medios en asignaturas de primaria como Lengua y Literatura, Conocimiento del Medio y algunas optativas en Secundaria y Bachillerato.

\subsection{El cuestionario}

Siguiendo la estructura de dimensiones indicadas para la medición de la competencia en comunicación audiovisual de Ferrés (2007: 100), se diseñó el instrumento principal que en este caso es el cuestionario. En la composición de cada uno de los ítems participaron las 17 universidades antes mencionadas que constituyó la parte inicial de la investigación general; luego, cada una de las comunidades autónomas podría analizar sus resultados. En Andalucía la prueba piloto se hizo efectiva en la provincia de Huelva con un mínimo de diez encuestas, con al menos un representante de cada una de las variables: provincia, edad, género y nivel de estudios. La validación fue precedida de las pruebas de control generales y la opinión de expertos metodólogos. El estudio de fiabilidad se realizó gracias al asesoramiento del Instituto de Ciencias de 
Educación (ICE) de la Universidad de Barcelona ${ }^{11}$. El cuestionario consta de 28 preguntas entre abiertas y cerradas. Los criterios de calificación se obtienen del trabajo de investigadores de la Universidad Pompeu Fabra y de los responsables de las áreas de educación artística del Departamento de Educación de la Generalitat de Catalunya.

\subsection{Las dimensiones analizadas}

\subsubsection{Dimensión estética}

El valor asignado a esta dimensión es de 15 puntos. Los contenidos que se consideraron en esta dimensión fueron: la innovación formal, la temática del producto analizado, la educación del sentido estético y la relación con otras formas de manifestación mediática y artística. Para medirla se propuso la valoración de dos anuncios publicitarios para que escogiera el mejor desde el enfoque artístico, argumentando las razones de su elección.

Los criterios de evaluación a esta pregunta fueron los siguientes: la máxima puntuación -15 puntos- si la persona justificaba adecuadamente su elección, es decir, además de hacer referencia al contenido y su realización, hace uso de adjetivo, y utiliza comparaciones para recordar otras experiencias similares; 10 puntos si argumentaba suficientemente pero sin comparar con otras experiencias visuales recibidas anteriormente; la persona obtendría 5 puntos si su justificación fura pobre y utilizara algún adjetivo. Como resultado se obtuvo una muy baja respuesta satisfactoria. Solo un $8.1 \%$ argumentó su elección utilizando varios adjetivos, haciendo referencias y asimilándola a alguna otra experiencia vivida. Un 49.7\% explicó el anuncio pero no argumentó efectivamente las razones de su elección, mientras que el $91.8 \%$ utilizó argumentos escasos y precarios. Los resultados generales figuran en la Tabla 2.

Tabla 2. Resultados obtenidos en la dimensión estética

\begin{tabular}{|c|c|}
\hline Por provincia & $\begin{array}{l}\text { Suspenden en los siguientes porcentajes: Sevilla }(100 \%) \text {, Málaga } \\
(97,4 \%) \text {, Huelva }(96,2 \%) \text {, Jaén }(95,5 \%) \text {, Almería }(94,8 \%) \text {, Cádiz } \\
(91,3 \%) \text {, Córdoba }(82,4 \%) \text { y Granada }(78,2 \%) \text {. }\end{array}$ \\
\hline Por género & $\begin{array}{l}\text { Un } 92.0 \% \text { de los hombres no justifican su respuesta apropiadamente } \\
\text { frente a un } 91.8 \% \text { de las mujeres. }\end{array}$ \\
\hline Por edad & $\begin{array}{l}\text { Los de la tercera edad fallan en un } 97.5 \% \text { y los jóvenes (15-24 años) les } \\
\text { siguen con un } 93.1 \% \text {. De los adultos (25-64 años) apenas el } 10.1 \% \text { se } \\
\text { muestra capaz de evaluar el mensaje desde esta dimensión. }\end{array}$ \\
\hline Por nivel de estudios & $\begin{array}{l}\text { Solo el } 14.6 \% \text { de los universitarios evalúa la dimensión estética ade- } \\
\text { cuadamente, mientras que quienes tienen estudios secundarios lo hacen } \\
\text { apenas en un } 6.7 \% \text {. Los que no tienen estudios en un } 3.4 \% \text { y sorpren- } \\
\text { dentemente solo un } 1 \% \text { de los que tienen estudios primarios, acierta. }\end{array}$ \\
\hline
\end{tabular}

${ }^{11}$ Cfr. El investigador en metodología cuantitativa Francesc Martínez realizó las revisiones de los cuestionarios antes de su aplicación y posteriormente validó los resultados en relación con los obtenidos en la prueba piloto. Algunas de las dimensiones presentaron ciertas dificultades a la hora de la validación pues en ocasiones la medición se realiza mediante una sola pregunta, lo cual imposibilita la fiabilidad. En otros casos, la multiplicidad de preguntas, unas genéricas y otras muy concretas en relación con una misma dimensión, trae como consecuencia la disparidad. La recomendación del investigador fue realizar el cuestionario sin especificar las dimensiones, compararlas ni relacionarlas entre sí. Sin embargo, para este estudio se conservó la estructura de las dimensiones puesto que forma parte del planteamiento base de la investigación. 
Se puede observar que los llamados "nativos digitales" no alcanzan mejores cifras que los adultos: se muestra capaz el $6.9 \%$ de los primeros frente al $10.1 \%$ de los segundos. Está claro que esta dimensión ha de evaluarse de modo más firme y riguroso. Es evidente que no existe formación estética en el área audiovisual, la escasa destreza para razonar por escrito y la ausencia de formación para consumir con criterio productos audiovisuales.

\subsubsection{Dimensión de lenguaje}

El valor asignado a esta dimensión es de 20 puntos. En esta ocasión se tomó en cuenta el conocimiento de los códigos necesarios para comprender el lenguaje audiovisual y la capacidad para utilizarlo de un modo simple. Sumado a ellos está el análisis desde la significación de las estructuras narrativas y de las categorías y géneros. Por ser una dimensión con varias aristas de interés es evaluada a través de distintas preguntas. Para valorar aquellas relacionadas con el conocimiento de los códigos se acordó seguir los siguientes criterios ante el visionado de un anuncio: 10 puntos si el sujeto menciona y justifica su respuesta utilizando códigos relacionados con la planificación, profundidad de campo, ángulos de cámara, color, ritmo del vídeo, etc. Si únicamente los menciona obtiene 5 puntos, y ninguno -0 puntos- si no hace ninguna referencia al lenguaje audiovisual. Otra pregunta requería que el sujeto eligiera una fotografía, de entre varias, que más se adecuara a un título dado. Al responder a la razón por la cual eligió la imagen se pretendía evaluar su capacidad de argumentación utilizando códigos del lenguaje audiovisual. Una cuestión distinta se pedía en otro ítem al solicitarle que construyera una historia coherente utilizando 6 imágenes dadas; solo la primera era inamovible. Se buscaba indagar si el sujeto conocía las reglas del lenguaje audiovisual -como el raccord por ejemplo- al formar dicha secuencia. Los resultados generales según las variables están plasmados en la Tabla 3:

Tabla 3. Resultados obtenidos en la dimensión de lenguaje.

\begin{tabular}{|l|l|}
\hline Por provincia & $\begin{array}{l}\text { Las siete provincias restantes obtienen el } 100 \% \text { de valoración negativa, } \\
\text { es decir, suspenden, excepto Cádiz (con un 98.1\%). }\end{array}$ \\
\hline Por género & $99.6 \%$ de los hombres suspenden frente a un $99.7 \%$ de las mujeres. \\
\hline Por edad & $\begin{array}{l}\text { Jóvenes y mayores obtienen por igual una valoración negativa del 100\%. } \\
\text { Solo en el caso de los adultos se reduce mínimamente esta cifra pues fa- } \\
\text { llan en un } 99.5 \% .\end{array}$ \\
\hline Por nivel de estudios & $\begin{array}{l}\text { Todos los niveles obtienen porcentajes negativos. Solo los sectores de } \\
\text { estudios secundarios y universitarios presentan una exigua diferencia, } \\
99.6 \% \text { y } 99.5 \% \text { respectivamente; los demás - sin estudios y con estudios } \\
\text { primarios- suspenden en un } 100 \% .\end{array}$ \\
\hline
\end{tabular}

Se puede considerar tajantemente que existe un conocimiento mínimo en esta dimensión del lenguaje audiovisual y además dificultad para expresarlo. Los resultados dejan entrever en el apartado de jóvenes -junto a los mayores- una incapacidad en la evaluación de esta dimensión del 100\%. El desconocimiento del lenguaje y la gramática audiovisual impide, por un lado, la lectura crítica del medio, y por el otro, su disfrute y aprovechamiento. 


\subsubsection{Dimensión de la ideología y de los valores}

Esta dimensión tiene un valor asignado de 25 puntos. En este caso se valora la capacidad de lectura comprensiva y crítica del mensaje. La identificación de ideologías y valores está repartida en diversas preguntas -cinco concretamente-, que a su vez evalúan otras dimensiones. Se otorgan 10 puntos a quien es capaz de captar todos los valores del anuncio visionado, 5 si solo da cuenta de algunos de los valores, y 0 puntos si no hace referencia a ninguno. En este caso se midieron las reacciones del encuestado ante un anuncio en relación con los siguientes aspectos: su conocimiento acerca de la transmisión de ideologías y valores; la credibilidad que la imagen otorga a la información; y las emociones, en tanto que está íntimamente relacionada con la toma de decisiones. Si analizamos la Tabla 4 con perspectiva, observamos los siguientes resultados:

Tabla 4. Resultados obtenidos en la dimensión de ideología y valores.

\begin{tabular}{|l|l|}
\hline Por provincia & $\begin{array}{l}\text { Obtienen valoraciones negativas todas las provincias pues suspenden } \\
\text { entre un } 89.9 \% \text { de Jaén a un } 97.7 \% \text { de Sevilla. }\end{array}$ \\
\hline Por género & $\begin{array}{l}\text { Muy igualados pues los hombres suspenden en un 94\% y las mujeres en } \\
\text { un 93\%. }\end{array}$ \\
\hline Por edad & $\begin{array}{l}\text { Valoración negativa en un } 92.1 \% \text { de los adultos (25-64 años), en un } \\
93.1 \% \text { de los jóvenes (16 a } 24 \text { años), mientras los que tienen más de } 64 \\
\text { años suspenden en su totalidad (100\%). }\end{array}$ \\
\hline Por nivel de estudios & $\begin{array}{l}\text { Respuestas incorrectas del } 90.4 \% \text { de universitarios, el 92.9\% de los que } \\
\text { tienen estudios secundarios mientras que el resto (estudios primarios y } \\
\text { sin estudios) no supera el } 1 \% \text { de valoración positiva. }\end{array}$ \\
\hline
\end{tabular}

Aquí se presenta una situación similar que en las dimensiones anteriores. La capacidad de lectura crítica es minúscula, apenas se distingue en un $7.1 \%$ de quienes tienen estudios secundarios y del $9.4 \%$ de los universitarios. Efectivamente parece haber una relación directa entre el nivel de estudios y su grado de reflexión en el visionado audiovisual, pero sigue siendo muy bajo el nivel. Prevalece el hecho de que algunos creen que las imágenes no pueden engañarle o que las emociones no influyen a la hora de tomar una decisión. La transmisión de ideologías y valores cuenta con mecanismos nada simples para lo cual parece no estar preparada la ciudadanía.

\subsubsection{Dimensión de la recepción y la audiencia}

En este caso el valor asignado a la dimensión es de 13 puntos. En ella se examina el conocimiento acerca del proceso de recepción del mensaje audiovisual y su percepción acerca de la medición de audiencias. El principio que procede del campo de la psicología y de la comunicación persuasiva conocido como "ilusión de invulnerabilidad", consiste en aceptar la fuerza y el poder de los medios de comunicación solo cuando se trata de grandes masas, pero no en el ámbito personal. En esta dimensión se evalúa este principio junto a otros aspectos como la interacción entre emociones y razonamientos, el conocimiento sobre el audímetro y la corresponsabilidad social como parte de la audiencia. La Tabla 5 muestra los resultados arrojados en relación con las variables positivas: 
Tabla 5. Resultados obtenidos en la dimensión de recepción y audiencia

\begin{tabular}{|l|l|}
\hline Por provincia & $\begin{array}{l}\text { Sevilla aprueba en un } 14 \%, \text { Huelva y Granada en 20.5\%, Almería y Cádiz } \\
\text { en } 22.1 \% \text {, Córdoba en } 27.5 \% \text {, Jaén en 31.5\% y Málaga en 38.5\% de los } \\
\text { encuestados. }\end{array}$ \\
\hline Por género & $\begin{array}{l}\text { Aprueban con gran similitud en los resultados un } 25.5 \% \text { de los hombres } \\
\text { y un } 25.1 \% \text { de las mujeres. }\end{array}$ \\
\hline Por edad & $\begin{array}{l}\text { Los jóvenes (16-24) aciertan en un } 20 \%, \text { los adultos (25-64) en un 31.6\% } \\
\text { y los de la tercera edad en un } 9.9 \% .\end{array}$ \\
\hline Por nivel de estudios & $\begin{array}{l}\text { Los universitarios aciertan en un } 36.5 \%, \text { los de secundaria en un } 23.4 \%, \\
\text { los que tienen solo estudios primarios en un } 14.7 \% \text { y los sin estudios en } \\
\text { un } 3.4 \% .\end{array}$ \\
\hline
\end{tabular}

En este caso a medida se observa que a medida que asciende el nivel de estudios, mejoran los conocimientos acerca de e la audiencia. Aún así son bajas las cifras pues se sitúan en un $36 \%$ en el mejor de los casos, lo que hace suponer que la colectividad ignora cuáles son sus derechos que tiene como audiencia y cuál es el alcance de su responsabilidad social.

\subsubsection{Dimensión de la tecnología}

Valor asignado: 15 puntos. De todas las dimensiones ésta es quizás la más conocida pues comprende el manejo de herramientas tecnológicas sencillas que tienen que ver con el consumo audiovisual. La temática fue abarcada por seis preguntas del cuestionario. Se pide información sobre conceptos tecnológicos tales como: memoria digital, You Tube, ipod, etc., conocimiento sobre el tratamiento tecnológico de la imagen, habilidades para encender o poner en funcionamiento un reproductor de DVD, detalles acerca del uso del teléfono a través de Internet y cuestiones sobre los derechos de autor. Los resultados acertados de esta dimensión, registrados en la Tabla 6, parecen destacar entre los demás:

Tabla 6. Resultados obtenidos en la dimensión de tecnología

\begin{tabular}{|l|l|}
\hline Por provincia & $\begin{array}{l}\text { Valoraciones positivas en las siguientes medidas: Sevilla } 46.5 \%, \text { Jaén } \\
50 \%, \text { Huelva } 53.3 \% \text {, Málaga } 55.6 \%, \text { Almería 57.1\%, Cádiz 60.5\%, Cór- } \\
\text { doba } 64.7 \% \text { y Granada } 77.3 \% .\end{array}$ \\
\hline Por género & $\begin{array}{l}\text { Muy igualados, } 59.9 \% \text { de los hombres aciertan y } 59.1 \% \text { de las mujeres } \\
\text { también. }\end{array}$ \\
\hline Por edad & $\begin{array}{l}\text { Aprueban los jóvenes (16-24) en un } 67.3 \% \text {, los adultos (25-64) en un } \\
63.8 \% \text { y los mayores de } 64 \text { años en un } 27.1 \% .\end{array}$ \\
\hline Por nivel de estudios & $\begin{array}{l}\text { Los universitarios presentan valoraciones positivas en un } 74.2 \%, \text { los de } \\
\text { secundaria en un } 60.9 \% \text {, con estudios primarios en un } 38.8 \% \text { y los sin } \\
\text { estudios en un } 26.9 \% .\end{array}$ \\
\hline
\end{tabular}

Se manifiesta una relación inversamente proporcional en cuando a la edad. A menor edad del sujeto, mayor conocimientos sobre tecnología. Todo lo contrario sucede en relación con la variable que clasifica al sujeto por su grado de preparación académica: a mayor nivel de estudios, mayor conocimientos sobre el tema tecnológico. Está claro que hasta este momento se trata de la dimensión mejor manejada por la muestra, aún 
cuando globalmente los resultados siguen siendo no muy altos, es decir, casi el 50\% de los individuos presentan escasa competencia en tecnología. El porcentaje más bajo se presenta en la variable edad relacionada con las personas mayores de 64 años.

\subsubsection{Dimensión de la producción y la programación}

Valor asignado: 12 puntos. Esta última dimensión evalúa sobre todo el conocimiento acerca de las rutinas de producción y organización necesarias para realizar un producto audiovisual. Se quiere conocer lo que sabe el sujeto en relación con el software libre, las profesiones vinculadas con la producción audiovisual, el proceso de producción en sí y las cuestiones vinculadas a la televisión pública y privada. Los resultados lanzaron los porcentajes que muestra la Tabla 7.

Tabla 7. Resultados obtenidos en la dimensión de producción y programación

\begin{tabular}{|l|l|}
\hline Por provincia & $\begin{array}{l}\text { No aprueban la dimensión el 82\% en Jaén, el 86.5\% en Cádiz, el 87\% en } \\
\text { Almería, el } 87.2 \% \text { en Granada, el 91\% en Huelva, el 70.5\% en Málaga } \\
\text { y el 78.4\% en Córdoba. }\end{array}$ \\
\hline Por género & Valoraciones negativas: $81.7 \%$ hombres, $86 \%$ mujeres. \\
\hline Por edad & $\begin{array}{l}\text { Los jóvenes }(16-24) \text { fallan en un } 86.2 \%, \text { los adultos destacan pues sus- } \\
\text { penden en un } 80.7 \% \text { y los de la tercera edad lo hacen en un } 95.1 \% .\end{array}$ \\
\hline Por nivel de estudios & $\begin{array}{l}\text { Suspende el } 75 \% \text { de universitarios, el } 85.9 \% \text { de quienes tienen estudios } \\
\text { secundarios, el } 93.1 \% \text { con estudios primarios y el } 96.6 \% \text { de las personas } \\
\text { sin estudios. }\end{array}$ \\
\hline
\end{tabular}

Al igual que en la dimensión tecnológica, el nivel de estudios presenta una relación directamente proporcional con el conocimiento evaluado. A mayor grado de preparación académica, mayor conocimiento de la dimensión. Un 25\% de los universitarios resulta aprobado, es decir, obtiene una puntuación superior al 50\% del total del valor asignado. Le siguen descendientemente los demás niveles: 14\% de los de secundaria, 6.9 de los de primaria y $3.4 \%$ de los sin estudios. En general, cifras con alta valoración negativa.

Si hacemos un recuento de los resultados del cuestionario podemos resumir que el grado de competencia audiovisual de los andaluces encuestados mayores de 16 años es muy bajo -un $97.9 \%$ obtiene valoraciones negativas- $\mathrm{y}$ apenas una persona alcanza la puntuación de 69 puntos sobre 100 , que continua siendo baja.

En cuanto a provincias, las tablas de contingencia de las puntuaciones

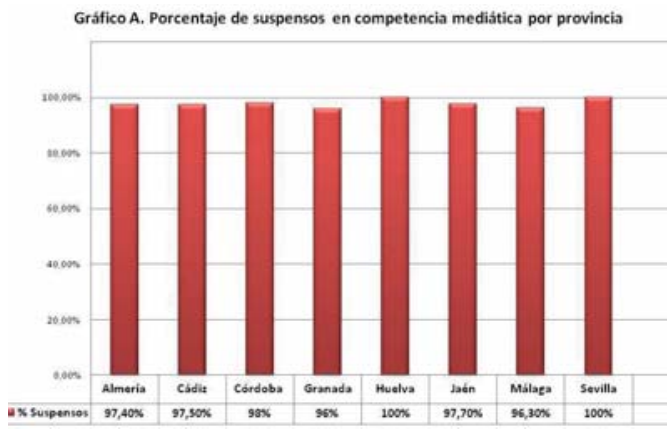
totales mostraron mayores valoraciones negativas en Huelva y Sevilla (100\%). En el gráfico A se observa que aunque la cifra desciende en el resto de las comunidades, lo hace en valores mínimos: Almería 97.4\%, Cádiz 97.5\%, Córdoba 98\%, Granada 96\%, Jaén $97.7 \%$ y Málaga 96.3\%. La analogía se presenta también en el caso de la obser- 


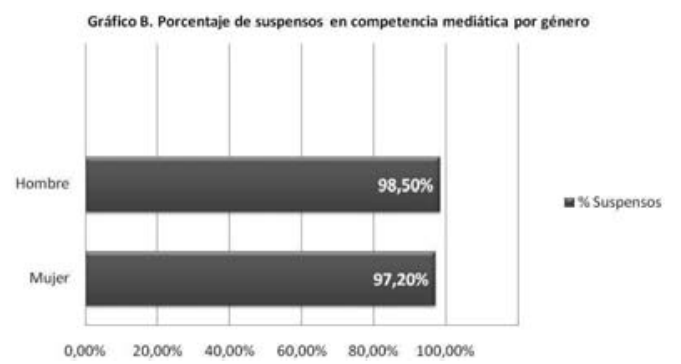

lo llevan indudablemente las personas mayores - 65 años ó más- con el 100\%, mientras que los adultos ( 25 a 64 años) se presentan como el segmento de mayor competencia en esta materia con un $97,1 \%$.

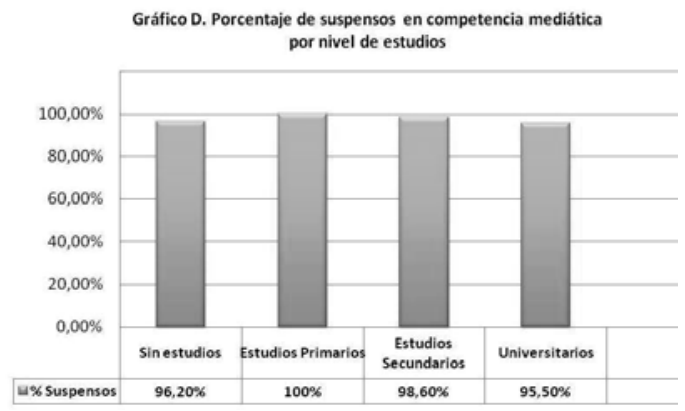

\section{Conclusiones}

Son múltiples las razones por las cuales se considera que una persona en el siglo XXI ha de estar preparada para interpretar los mensajes de los medios de comunicación que se expanden en el mundo digital. La Asociación para la Educación Mediática de Canadá -Association for Media Literacy (AML)- afirma que para que esto se consiga el individuo ha de conocer los distintos lenguajes de los medios, los estilos, las técnicas, los códigos y convenciones y también la estética, debe saber en qué consiste la transmisión de mensajes ideológicos y de valor de estos mensajes que pueden abarcar áreas políticas, sociales y comerciales, entre otros aspectos importantes ${ }^{12}$. La competencia en comunicación audiovisual, que no es más que la capacidad para interpretar mensajes audiovisuales de manera crítica y reflexiva y para expresarse a través del lenguaje audiovisual con unos mínimos de corrección y de creatividad, no se forma de manera espontánea por mucho que se hable de los nativos digitales. Es el resultado de acciones educativas precisas -ausentes hasta el momento- que han de implementarse lo antes posible.

Estamos ante unos resultados muy bajos en la competencia mediática medida en la ciudadanía andaluza. Las razones de éstos escapan al objetivo de este trabajo. No

12 Cfr. El portal puede consultarse en: www.aml.ca/whatis. 
obstante, si se exploran los resultados de las seis dimensiones que componen la competencia mediática, y que han sido aplicadas en este estudio -estética, lenguajes, ideología y valores, tecnología, producción y programación, y recepción y audiencia- se puede concluir que esas carencias pueden surgir desde distintos puntos que en conjunto abarcan a la familia, la escuela, las políticas y los propios medios de comunicación. Pero es conveniente describir esos resultados. En relación a la dimensión estética, los encuestados mostraron escasa habilidad para valorar el producto audiovisual que se les mostró. Las respuestas estaban salpicadas de generalizaciones sin mayores explicaciones que dieran sentido a este tipo de conocimiento: cómo ha sido realizado, uso de adjetivos y comparaciones, referencia a experiencias visuales ya conocidas, etc. La segunda dimensión, la del lenguaje, tampoco estaba inmersa en el intelecto de los entrevistados. No reconocieron los códigos ni fueron capaces de explicar visualmente, de manera adecuada, una historia a partir de las imágenes dadas.

En cuanto a la identificación de ideología y valores, se observa un esfuerzo por lograrlo, aún cuando no lo consiguen si no están expresamente manifiestos en el producto audiovisual. Lo mismo ocurre con la credibilidad ante las imágenes. No se percatan de la posible manipulación de la imagen sino que les otorgan un grado alto de credibilidad. Desconocen si los contenidos intervienen en sus propias emociones o cómo gestionarlas. Lo relacionado con la recepción y audiencia muestra la incapacidad para distinguir entre las emociones y los argumentos. Este estudio confirma la presencia en la muestra del principio de invulnerabilidad, en el cual la mayoría piensa que los mensajes no le influyen en lo personal pero sí afectan a los demás. Se observa, por otro lado, que los individuos desconocen a quien acudir -o cómo hacerlo- en caso de manifestar una queja o desacuerdo con algún medio o contenidos de los medios de comunicación.

La única dimensión que resulta medianamente favorecida en los resultados es la tecnológica. En cuando a temas relacionados con definiciones y manejo de la tecnología audiovisual, los encuestados superan ciertos niveles, aunque tampoco se pueden considerar satisfactorios. Los integrantes del segmento de mayor edad -más de 65 años- no son muy diestros ante dichos dispositivos o procesos tecnológicos, así como tampoco acerca de los derechos de autor. La última de las dimensiones, la de producción y programación, muestra al igual que las primeras analizadas, muy bajo nivel de conocimiento. Aspectos relativos a la propiedad intelectual en Internet, las similitudes y diferencias entre la televisión pública y la privada o los propios procesos de producción de un producto audiovisual son desconocidos a ciencia cierta por la mayoría.

En general, en cuanto a la clasificación de los resultados por provincia se evidencia una mínima diferencia entre cada una de las ocho que conforman la comunidad autónoma andaluza. En todas se obtienen altas valoraciones negativas, con Sevilla con la más baja puntuación y Granada con la mayor. Igual situación se presenta en relación con el género. Ambos fallan, ellos en un $98.5 \%$ y ellas en un $97.2 \%$. La edad refleja una relación inversamente proporcional en la mayoría de dimensiones: a mayor edad, menor competencia mediática, sobre todo en el rango de 65 años ó más. El nivel de estudios presenta una situación aparentemente lógica -aunque no concluyentecomo lo es el hecho de que a medida que aumenta el nivel de estudios, se manifiesta un mayor porcentaje de valoraciones positivas. El matiz lo marca el hecho de que los 
jóvenes y adultos con estudios secundarios y universitarios fallaron más en porcentaje que los de la tercera edad con iguales estudios.

A juicio de los integrantes de este grupo de investigación, y siguiendo las recomendación del Parlamento Europeo (2008), sería necesaria la integración de la asignatura educación mediática o educación en comunicación audiovisual (ECA), en el currículo escolar de Primaria y Secundaria y también hacer accesible la alfabetización mediática de adultos. El modo de implementación de esta asignatura requeriría de un estudio pormenorizado de cada nivel de enseñanza: diseño de un marco conceptual y de objetivos con sus correspondientes estrategias a lograr desde Infantil hasta Secundaria. En todo caso, la educación en comunicación mediática debe abordarse en primer lugar desde las seis dimensiones que otros estudios antes realizados han aplicado en relación con la competencia en comunicación audiovisual. La presente investigación demuestra que la población encuestada -que constituye la muestra- carece de toda competencia en dichas dimensiones. En segundo lugar, se ha de tener en cuenta la competencia emocional dada la vinculación de la imagen -fija o en movimiento- con el mundo de las emociones y del inconsciente. También debería de estudiarse el principio social de responsabilidades compartidas, término comúnmente utilizado en el campo medioambiental, en el entorno de la competencia mediática. La participación de distintos colectivos sociales reforzaría la solución a la insuficiencia demostrada en este estudio, como son por ejemplo los profesionales de la comunicación audiovisual, los de la enseñanza obligatoria, los formadores universitarios, y por supuesto, el núcleo familiar de padres, madres y abuelos, quienes con su dosis de responsabilidad y acercamiento a sus hijos pueden lograr mucho más. Cada uno ha de aportar en su medida, detectando los problemas, aportando experiencias, analizando los planes de estudios, seleccionando y evaluando las "buenas prácticas" que ya existen en este campo y formando a los docentes, es decir, transformándose en multiplicadores de la acción educativa en competencia mediática.

Como colofón, hay que recordar que la educación para los medios nace con una visión amplia y profunda, una perspectiva multidisciplinar que favorece a otras competencias desde el enfoque de los valores humanos.

La educación en medios tiene el potencial de reducir las desconexiones entre viejos y nuevos medios de comunicación, alta y baja cultura, medios patentados y no patentados, conflictos culturales y comerciales, etc. Ofrece un escenario para la sostenibilidad, sobre todo con recursos compartidos e iniciativas abiertas. Por último, puede ayudar a lograr los objetivos de la Declaración del Milenio, sobre todo, la erradicación de la pobreza y el analfabetismo (Frau-Meigs y Torrent, 2009: 14).

Torrent y Frau-Meigs (2009) proponen además el canon de las seis "C" de competencia clave en educación en medios, a saber: comprensión, capacidad crítica, creatividad, consumo, ciudadanía y comunicación intercultural.

\section{Agradecimientos y apoyos}

Estudio enmarcado en la Convocatoria de Proyectos I+D del Ministerio de Economía y Competitividad con clave: EDU2010-21395-C03-03, titulado "La enseñanza obligatoria ante la competencia en comunicación audiovisual en un entorno digital" y en 
el Proyecto de Investigación de Excelencia de la Junta de Andalucía SEJ-5823-2010, denominado "La competencia audiovisual de la ciudadanía andaluza. Estrategias de alfabetización mediática en la sociedad del ocio digital".

\section{Referencias bibliográficas}

ASSOCIACION FOR MEDIA LITERACY (Ed., 2011): "What is Media Literacy". (www.aml.ca/whatis) [Consultado: 16-08-2011]

FERRÉS, Joan (2007): "La competencia en comunicación audiovisual: dimensiones e indicadores". Comunicar, 29, XV, p. 100.

FERRÉS, Joan et al. (2011): Competencia mediática. Investigación sobre el grado de competencia de la ciudadanía en España. Madrid, Ministerio de Educación, ITE. (http://ntic.educacion.es/w3//competencia_mediatica/3_evaluacion/7.htm) ) [Consultado: 20-03-2012]

FRAU-MEIGS, Divina y TORRENT, Jordi (2009): “Políticas de educación en medios: Hacia una propuesta global". Comunicar, 32, p. 14.

ONU (Ed., 2012): Media Literacy. New York, Alianza de las Civilizaciones (www.aocmedialiteracy.org/index.php?lang=spanish) [Consultado: 22-02-2012]

PIETTE, Jacques (2009): “The challenges of media literacy. Euromeduc: A European exchange network for media literacy". Bruxelles, 115-124. http://www.euromeduc.eu/IMG/pdf/Euromeduc_ENG.pdf. [Consultado: 16/03/2012]

PARLAMENTO EUROPEO (Ed., 2006): Recomendación del Parlamento Europeo y del Consejo de 18 de diciembre de 2006 sobre las competencias clave para el aprendizaje permanente (2006/962/CE). Bruxelles, European Parlament (http://eur-lex.europa.eu/LexUriServ/LexUriServ.do?uri=OJ:L:2006 :394:0010:0018:es:PDF) [Consultado: 16-08-2011]

PARLAMENTO EUROPEO (Ed., 2009): Recomendación sobre la alfabetización mediática en el entorno digital para una industria audiovisual y de contenidos más competitiva y una sociedad del conocimiento incluyente. Bruxelles, European Parlament, p. 6. [Consultado: 16-05-2012] (http://ec.europa.eu/culture/media/lite$\mathrm{racy} / \mathrm{do})$.

PARLAMENTO EUROPEO (Ed., 2009): Un planteamiento europeo de la alfabetización mediática en el entorno digital. En: http://europa.eu/legislation_summaries/information_society/strategies/124112_es.htm [Consultado: 22-02-2012]:

PARLAMENTO EUROPEO (Ed., 2008): El PE sugiere introducir una asignatura de "educación mediática" en las escuelas europeas. http://www.europarl.europa.eu /sides/getDoc.do?language=es\&type=IM-PRESS\&reference=20081216IPR44614 Bruxelles, European Parlament [Consultado: 16-08-2011] 